\title{
Power-Flow-Optimising-Algorithm used in an Energy Management for Micro Grids
}

\author{
J. Teuscher ${ }^{1}$, B. Born ${ }^{1}$ and W. Schufft ${ }^{1}$ \\ ${ }^{1}$ Chair of Power and High-Voltage Engineering \\ Faculty of Electrical Engineering and Information Technology \\ Chemnitz University of Technology \\ Straße der Nationen 61, 09107 Chemnitz (Germany) \\ Phone/Fax number: +0049 371531 37752/+0049 371531 837752, e-mail: jens.teuscher@etit.tu-chemnitz.de
}

\begin{abstract}
This paper contains an overview about a powerflow-optimising-algorithm and the usage in an energy management for smart low-voltage grids (micro grids). This algorithm is based on a clustering of a number of households to reduce the distance between consumers and suppliers and so to reduce the transport losses in low-voltage grids. Such a power flow optimising enables to accomplish the requirements on lowvoltage grids (f.e. a high number of photovoltaic systems or electric vehicles). Furthermore, the paper contends the usage of this algorithm in an energy management system and the results of some first calculations.
\end{abstract}

\section{Key words}

smart grid, micro grid, low-voltage grid, power flow simulation

\section{Introduction}

In the last years the energy system in Germany is in a difficult structural change. There are some economical, political and environmental challenges which are not solved yet. One of these challenges is the change from fossil energy sources to renewable energy sources for the supply of electrical energy. The government of Germany has the aim to supply at least $30 \%$ of the consumption of electrical energy with renewable energy sources in 2030. [1] For this challenge it is important to fulfil different tasks. The main task is the integration of a high number of decentralised power supply units, which are based on renewable energy sources (f.e. photovoltaic systems, hybrid storage systems with combined heat-powersystems).

The main focus for the integration of systems based on renewable energy sources is on the low-voltage grids. A standard low-voltage grid has the historical function to supply electrical energy from the transformer to a number of households or small industry. A high number of decentralised power supply systems will change this classical power flow in low-voltage grids. At the moment low-voltage grids are "black boxes" for the distribution systems operator and the power flow is unknown. For the development of energy managements it is very important to know the power flow in low-voltage grids.

For that reason one part of this paper is the simulation of the power flow in low-voltage grids with fluctuating consumptions and supplies. Furthermore, the paper gives an overview about a management solution and the powerflow-optimising-algorithm, which is based on building different groups (clustering) in low-voltage grids.

\section{Overview about Management Solution}

A high number of decentralised power supply systems in low-voltage grids have different consequences. On the one hand the limitation of the lines and the transformer must be observed. On the other hand the voltage drop has to be less than $10 \%$ in both directions. A management system has the goal to control these effects.

There are different solutions developed, which are based on the utilization of the lines or the transformer or which are based on the voltage drop. But one another main challenge are the transport losses in the low-voltage grids. The low-voltage grids cause the most transport losses (more than $40 \%$ ) in the electrical energy system [2]. If the reference area is reduce to small consumers (households, small industry), the transport losses for this small consumers are over $75 \%$ in low-voltage grids. The increasing of the number of decentralised power supply systems and also new types of consumers (f.e. electric vehicles) will cause an increasing of the transport losses in low-voltage grids.

For that reason it is very important to manage the power flow in these grids. This strategy has on big advantage. The power flow optimising includes the control of the voltage drop and the utilization of lines and transformer. Figure 1 gives an overview about a management solution to manage all conditions. This management strategy is divided in two parts. The first stage is the house management. It manages different storage systems (f.e. full cells, batteries, thermal storage) supply units consumers in one house (smart house). The second stage 


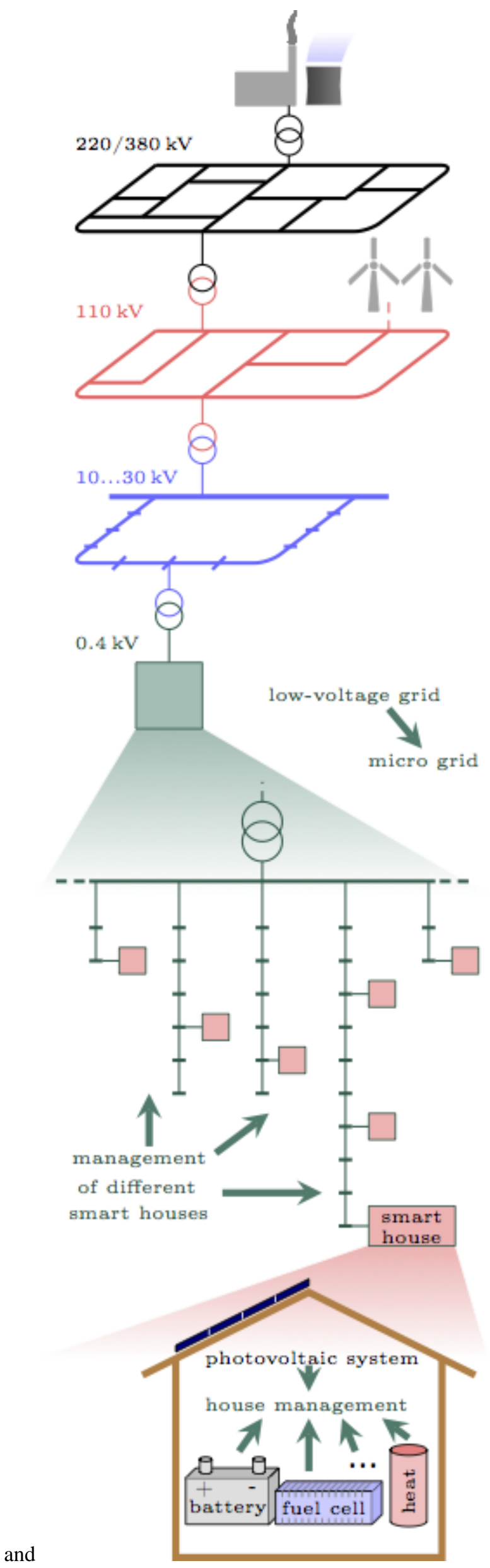

Fig 1. Overview of a management solution is the grid management, which oversee these smart houses in a low-voltage grid and the connection to the medium-voltage grid. The focus of this paper is on the management of such a micro grid.

One advantage of such an energy management with to stages is the different usage of the storages with two purposes. On the one hand the smart house manages the individual supply and consumption in the house. On the other hand the grid management control the voltage drop or the utilization of lines and transformer.

The connection between these two management systems is based on an incentive signal. Table I shows the incentive signal and the possible reaction of the smart houses.

Table I. - Incentive signal and the reaction of the smart houses

\begin{tabular}{|c|c|}
\hline $\begin{array}{c}\text { INCENTIVE SIGNAL } \\
\kappa\end{array}$ & $\begin{array}{c}\text { POSSIBLE REACTION OF THE } \\
\text { SMART HOUSES }\end{array}$ \\
\hline$\kappa<0$ & $\begin{array}{c}\mathrm{P}_{\text {house }} \text { decrease (more supply or less } \\
\text { consumption) }\end{array}$ \\
\hline$\kappa>0$ & $\begin{array}{c}\mathrm{P}_{\text {house }} \text { increase (more consumption or } \\
\text { less supply) }\end{array}$ \\
\hline$\kappa=0$ & $\mathrm{P}_{\text {house }}$ is constant \\
\hline
\end{tabular}

But this is only a possible reaction. The smart houses get only an incentive to react. The reaction of the smart houses depends on the actual individual load situation and on the possible option, f.e. available storage capacity. More details about the definition of the incentive signal and the usage are explained in section 4.

\section{Simulation of Micro Grids}

The basic for the development on grid management with power flow optimising is a simulated low-voltage grid. Low-voltage grids are at the end of the classical electrical energy system and supply small consumers. The topologies of these grids are different and depend on the geographical conditions. So it is not possible to modelling an universal low-voltage grid. Table II shows the typical differences between an urban low-voltage grid and a rural low-voltage grid.

Table II. - Differences between rural and low-voltage grid

\begin{tabular}{|l|c|c|}
\hline \multicolumn{1}{|c|}{ PARAMETER } & URBAN GRID & RURAL GRID \\
\hline $\begin{array}{l}\text { distribution } \\
\text { transformer capacity } S_{r}\end{array}$ & $630 \mathrm{kVA}$ & $250 \mathrm{kVA}$ \\
\hline Max. line length & $200 \mathrm{~m}$ & $1400 \mathrm{~m}$ \\
\hline $\begin{array}{l}\text { number of powered } \\
\text { households }\end{array}$ & 860 & 230 \\
\hline
\end{tabular}

The simulated low-voltage grids are based on these facts. In this paper the energy management is tested on a model of an urban low-voltage grid including almost 80 connection points over an area of $0.25 \mathrm{~km}^{2}$. The used simulating program is Matlab Matpower with some own developed extensions [3].

The challenge by simulating low-voltage grids is on the simulation different fluctuated load profiles. For the simulation of the consumption of different households a behaviour based load profile generator is used [4]. This 
generator simulates different load profiles, which depend on the number of people in a household, the different behaviour of the people and some other facts.

Figure 2 shows the comparison between a measured and a simulated load profile at the transformer station in a model.

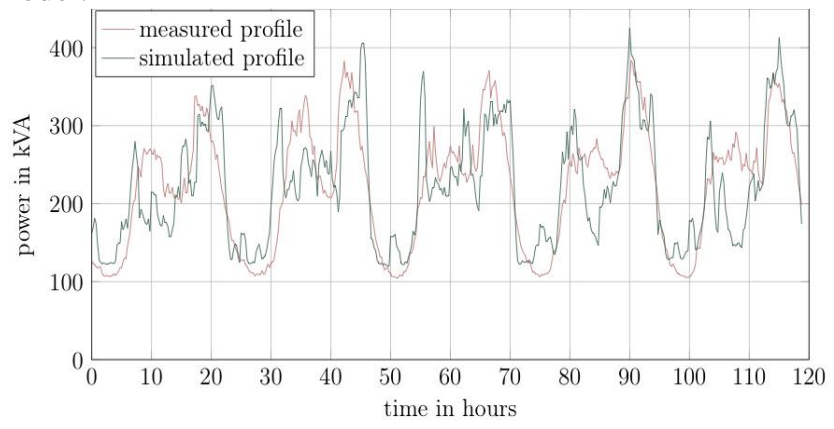

Fig 2. Comparison between measured and simulated load profiles at the transformer station in the model

This comparison shows the typical daily load profile in both curves. There are only some differences between the fluctuations, which will improve by the next development stage. With the usage of the load profile generator it is possible to simulate the fluctuations in the grid and modelling a high number of different load profiles in one grid, which includes a high relation to reality.

\section{Cluster-based Power Flow Optimising}

The power flow optimising is based on a clustering. The reason for that is to reduce the distance between consumption and supply and so to reduce the transport losses and the voltage drop. The clustering is based on the graph theory. For that reason the smart houses are called nodes in the following description.

The creation of groups is based on a bottom-up-principle, which is shown in figure 3 .

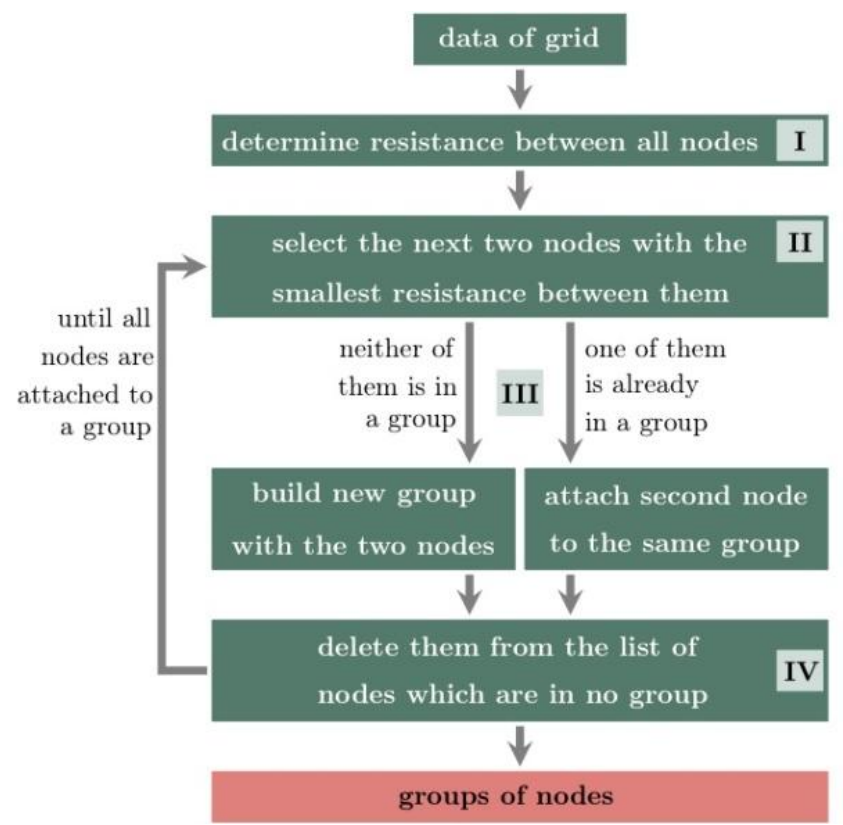

Fig. 3. Overview of building groups (clustering)

The considered parameter for clustering is the distance between the nodes. It starts with the smallest resistance and building different groups. This clustering is only realized at the beginning of the power-flow-optimising and is only one small part of the incentive signal for each node.

Figure 4 gives an overview about the different stages to create the incentive signal for each node.

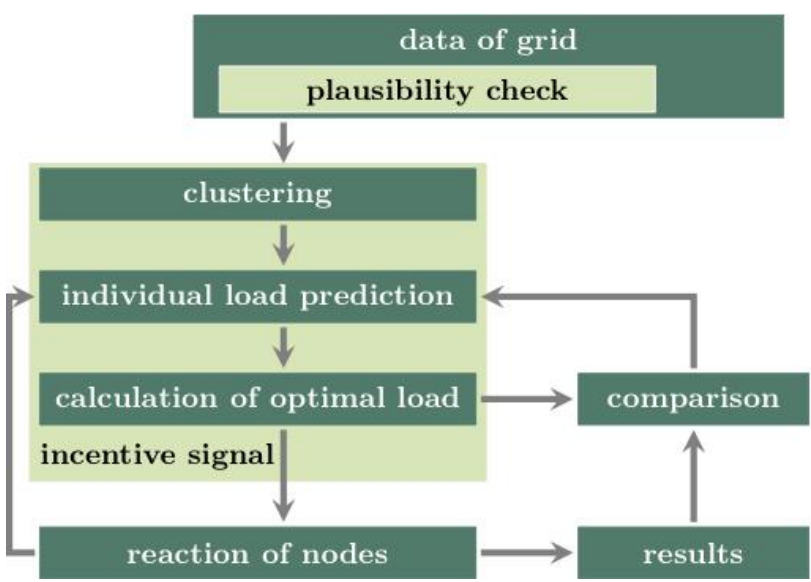

Fig. 4. Overview to create the incentive signal

The structure of the incentive signal is divided in different stages. Among the clustering is an individual load prediction of each node important. This prediction is based on the behaviour of the node in the past and some limiting parameters like the maximal load or the maximal change of the load. This prediction is compared with the real existing load at the nodes and the reaction of the nodes to the incentive signal. If one node is not reacting, the incentive signal is increasing for this node and his neighbour nodes.

\section{First Results of the Power Flow Optimising}

The result of the clustering in the simulated low-voltage grid is a structure of 22 different groups. Each group consists of 2-7 nodes. In the most cases one group complies one part of a path in the low-voltage grid.

The first goal of the algorithm is to balance the power flow in one group. Figure 5 shows this balancing in one group over some time steps for a test scenario.

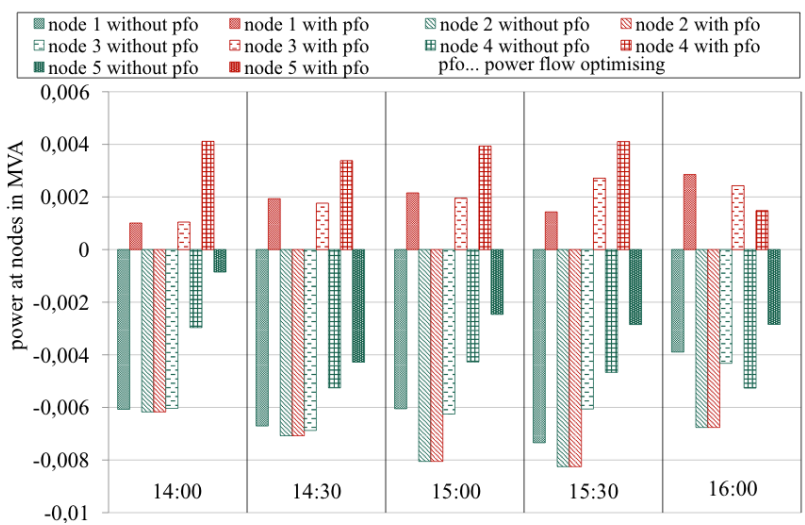

Fig. 5. Balancing in one group with power flow optimising

This test scenario is based on some border conditions. So the goal of the low-voltage grid is an autarkic grid operation independent on the medium-voltage grid. The 
load situation in this test scenario is a summation of the consumption of the households and a high supply by photovoltaic systems. Furthermore, every node can be a supplier or a consumer in the case of power flow optimising.

The green bars in figure 5 shows the load situation in the low-voltage grid without the power flow optimising. There is every node in the group a supplier, because the photovoltaic systems produce more electrical energy than the households are consumed.

The red bars contents the power flow optimising. It shows the balancing of the power flow in one group.

The results of the whole low-voltage grid for this test scenario are shown in figure 6.

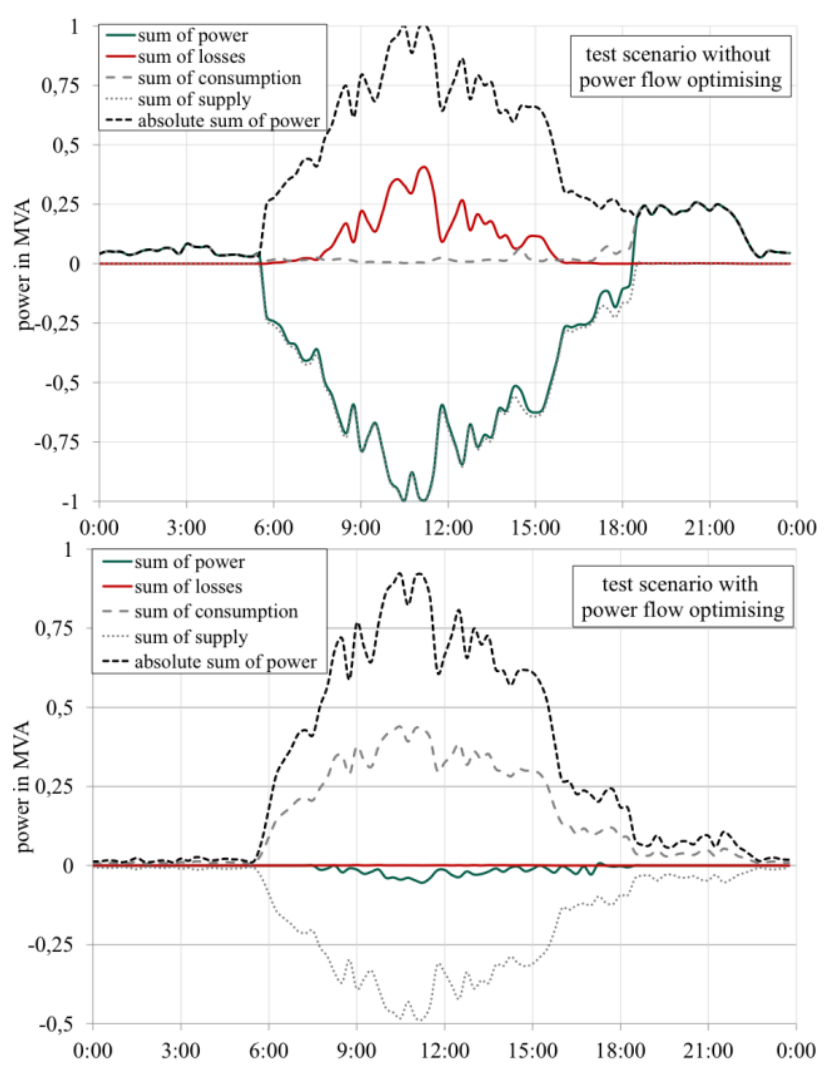

Fig. 6. Load profiles and losses dependent on the power flow optimising

The figure at the top shows the situation in the low-voltage grid without the power flow optimising. This is the case of a high supply with photovoltaic systems, which shows the green curve (sum of power). This case is not possible, because it breaks the limits of the utilization of the transformer and the limits of the voltage drop.

In the bottom of figure 6 is the same scenario with the power flow optimising illustrated. The goal of the optimising is an autarkical grid operation, which is shown in the green curve (sum of power). Furthermore, this figure shows the reduction of the supply by photovoltaic systems and the increase of the consumption in the low-voltage grid. With this power flow optimising it is possible to reduce obviously the transmission losses in the lines. In table III is a comparison of this test scenario between the grid operation with and without the power flow optimising based on the electrical energy per day stated.
Table III. - Differences between with and without power flow optimising for the test scenario

\begin{tabular}{|l|c|c|}
\hline \multicolumn{1}{|c|}{ PARAMETER } & $\begin{array}{c}\text { WITHOUT } \\
\text { PFO }\end{array}$ & WITH PFO \\
\hline $\begin{array}{l}\text { sum of consumption } \\
\text { per day }\end{array}$ & $301 \mathrm{kWh}$ & $157 \mathrm{kWh}$ \\
\hline sum of supply per day & $61 \mathrm{kWh}$ & $149 \mathrm{kWh}$ \\
\hline sum of losses per day & $62 \mathrm{kWh}$ & $0.3 \mathrm{kWh}$ \\
\hline
\end{tabular}

With this algorithm it is possible to reduce the power flow in the low-voltage grid and manage a high number of decentralised power supply systems in a micro grid. Furthermore, it is possible to connect a high number of decentralised power supply systems in a low-voltage grid without any breaks of the voltage drop or the utilization of lines and transformer.

Concerning the connection to the medium-voltage grid it is shown, that individual schedule of supply or consume is possible.

\section{Conclusion}

The paper shows the first steps of a cluster-based power flow optimising and the usage in a management for micro grids. The simulation of low-voltage grids with the fluctuations in consumption and supply are shown. So the basic for a power flow optimising is done.

Furthermore, the paper contents the structure of clustering and the integration in a grid management with the incentive signal.

The first calculations show an efficient application of the power-flow-optimising-algorithm to reduce the transport losses and get a high number of decentralised power supply systems in a low-voltage grid. But the management system is not sufficiently tested.

In the next steps the management is testing in different scenarios, which includes different typical low-voltage grids and different load situations of smart houses. Furthermore, the management is testing with a high number of none reaction of the nodes and the option to implement a grid operation with a individual schedule to supply or consume in comparison with the mediumvoltage grid.

\section{References}

[1] Federal Ministry for Environment, Nature Conservation and Nuclear Safety (BMU), "Energy Concept for an Environmentally Sound, Reliable and Affordable Energy Supply", German Government 28.10.2010, Berlin (2010)

[2] Federal Network Agency for Electricity, Gas, Telecommunications, Post and Railway, "MonitoringBericht 2012”, 3. Edition - 05.02.2013, Bonn (2013)

[3] R.D. Zimmermann, C.E. Murillo-Sánchez, "MATPOWER 4.1 User's Manual", PSERC (Power Systems Engineering Research Center), Tempe (2011)

[4] Pflugradt, N.; Platzer, B., "Behavior based load profile generator for domestic hot water and electricity use", Innostock, 12th International Conference on Energy Storage, Lleida (2012) 